\title{
Process Mining of Disease Trajectories: A Feasibility Study
}

\author{
Guntur P. Kusuma ${ }^{1,2} \mathbb{C}^{\mathrm{a}}$, Samantha Sykes ${ }^{3} \mathbb{C}^{\mathrm{b}}$, Ciarán McInerney ${ }^{1} \mathbb{( \mathbb { D }}^{\mathrm{c}}$ and Owen Johnson ${ }^{1} \mathbb{C}^{\mathrm{d}}$ \\ ${ }^{1}$ School of Computing, University of Leeds, Leeds, U.K. \\ ${ }^{2}$ School of Applied Science, Telkom University, Bandung, Indonesia \\ ${ }^{3}$ School of Medicine, University of Leeds, Leeds, U.K.
}

Keywords: Disease Trajectories, Process Mining, Electronic Health Records.

\begin{abstract}
Modelling patient disease trajectories from evidence in electronic health records could help clinicians and medical researchers develop a better understanding of the progression of diseases within target populations. Process mining provides a set of well-established tools and techniques that have been used to mine electronic health record data to understand healthcare care pathways. In this paper we explore the feasibility for using a process mining methodology and toolset to automate the identification of disease trajectory models. We created synthetic electronic health record data based on a published disease trajectory model and developed a series of event log transformations to reproduce the disease trajectory model using standard process mining tools. Our approach will make it easier to produce disease trajectory models from routine health data.
\end{abstract}

\section{INTRODUCTION}

Diseases occur at various points during a person's life-course and impact on health, lifestyle, quality of life, morbidity and mortality. Disease can be seen as a pathological process that requires judgement from a clinician to objectify its occurrence (Boyd, 2000). The record of disease occurrences over time become the "footprints" that can tell the story of how diseases have progressed for each individual. This type of historic patient information is vital evidence that can help clinicians to diagnosis appropriately and to decide on appropriate interventions (Muhrer, 2014; World Health Organization, 2016). More generally, medical research recognises common patterns of diseases where one disease is often found to precede others. These commonly-found patterns for disease progression are sometimes referred to as disease trajectories (A. B. Jensen et al., 2014).

The temporal record of diseases can be observed within electronic healthcare records (EHR) and can be used to understand the occurrence and behaviour of diseases. The trajectories of diseases can be identified by observing the sequence of disease diagnoses and the time intervals between them. Investigating disease trajectories has the potential to provide personalised medical treatment (P. B. Jensen et al., 2012) and to understand the potential causeand-effect association between diseases (Hanauer \& Ramakrishnan, 2013; Rothman \& Greenland, 2005).

In A. B. Jensen et al.'s (2014) widely cited work, the authors produced a number of disease trajectories based on EHR data from the population of Denmark. Disease trajectories were defined as the time-ordered sequence of diagnoses observed in the patients. An example of a disease trajectory model is presented in Figure 1. The model consist of nodes representing the diseases and directed arcs representing the common trajectories between diseases with the thickness of the arcs representing the relative number of patients.

In many countries, healthcare providers are now supported by EHR systems containing episodic and longitudinal data of a patient's medical history, diagnosis and treatment (Hemingway et al., 2018). The World Health Organisation had introduced standards for medical records (World Health Organization, 2002) and the European Medicines Agency suggests that clinicians use of medical record information is good clinical practice (European

\footnotetext{
a(D) https://orcid.org/0000-0002-0208-125X

b (iD https://orcid.org/0000-0001-7098-3928

c (iD) https://orcid.org/0000-0001-7620-7110

d(D) https://orcid.org/0000-0003-3998-541X
} 


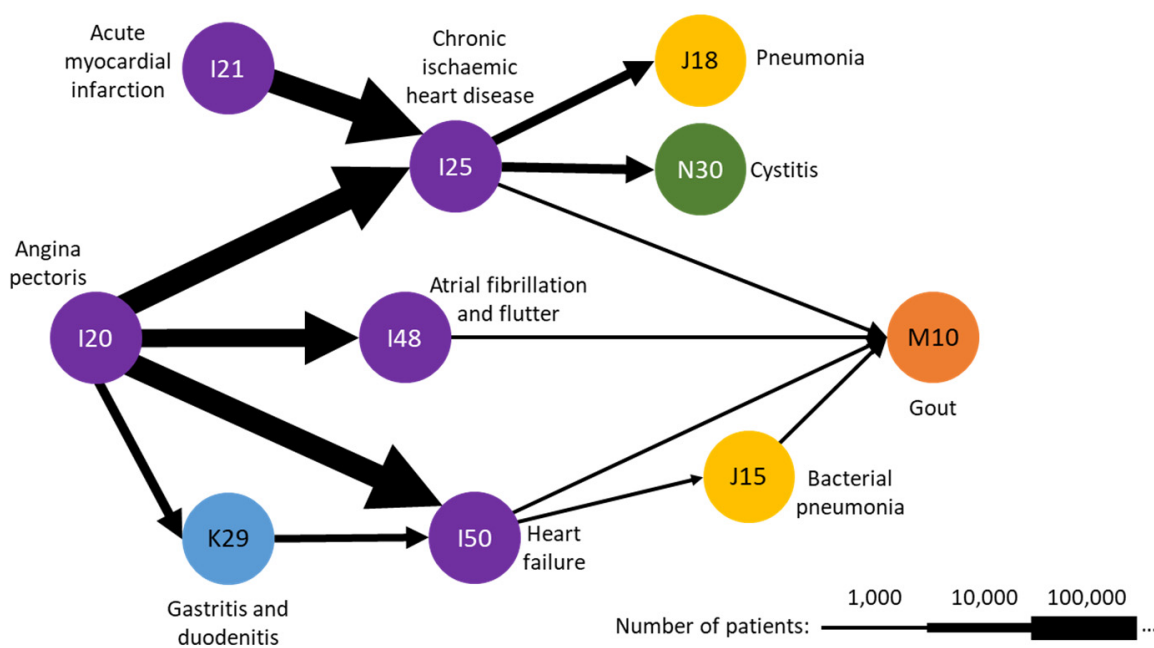

Figure 1: Example of a disease trajectory model, adapted from Figure 4.b of A.B. Jensen et al. (2014).

Medicines Agency, 2002). In some countries, the initial motivation of developing EHR was for billing purpose but in many countries EHR use is now comprehensive and includes records of disease diagnoses, with use being expanded for clinical and research purposes (Casey et al., 2016). The World Health Organisation provides the International Classification of Diseases (ICD) with the purpose of standardising the coding of diseases within EHR to support evidence-based decision making, sharing and comparing of health information, monitoring the incidence of disease, and helping healthcare organisations in managing disease related billing (WHO, 2019). Disease codes in EHR are commonly used, but there are known data quality issues. For example, in the ICD-10 standard, the code I20 is used for angina pectoris. This structured encoding framework has facilitated the construction of disease trajectories using EHRs (A. B. Jensen et al., 2014) and for process mining of care pathways (Rojas et al., 2016).

Process mining is a data mining approach that examines temporal and sequential data to analyse processes including the discovery of process models, conformance checking and process enhancement (van der Aalst, 2011). Process mining provides a holistic view, end-to-end analysis, and generates easy-to-read models and simulations (van der Aalst, 2011). The input of process mining is an event log detailing who did what and when. More formally, the event $\log$ is a collection of time stamped events containing at least a case, an activity, and a timestamp. The output of process mining is often graphical, producing visual models of processes and pathways. Conformance of event logs to expected models can be measured and a process modelling project may involve multiple iterations of data extraction, transformation, modelling, measuring and refinement to construct valuable process models and process insights.

In our review of the literature, we found that process mining techniques have not yet been utilised to extract patients' disease trajectory models from health data despite the many similarities between process models and disease-trajectory models. This is despite the data required for such an approach often being available within EHRs.

The aim of this study, therefore, was to assess the feasibility of using process mining methods and tools with EHR data to construct disease trajectory models. In our study, we simulated a scenario from A. B. Jensen et al.'s trajectories that were centred on chronic ischaemic heart disease (the example in Figure 1). Our hypothesis was that by treating the entry of a disease code in the EHR as the equivalent of an activity in process mining we could exploit the rich toolset of process mining for the mining of disease trajectories.

\section{BACKGROUND}

\subsection{Disease Trajectory}

\subsubsection{Definitions}

Multiple definitions of disease trajectory have been proposed. Murray et al (2005) defined a disease trajectory as the progressiveness of physical health deterioration over time. They described three types of trajectories: the short period, where the decline of physical health happens within a few months or a few 
years; long-term limitations, where the decline happens between 2 to 5 years; and prolonged dwindling, where the decline happens in 6 to 8 years. A disease trajectory is also defined as the progression of a specific disease by observing a clinical measure of the severity of a disease. An example of work which follow this definition is a study observing the progression of chronic kidney disease by measuring the estimated glomerular filtration rate (eGFR) (Sumida \& Kovesdy, 2017). Finally, A. B. Jensen et al. (2014) proposed a definition of disease trajectory as the sequence of diseases that are ordered by the time of the occurrence. Our work follows this definition from A. B. Jensen et al. We note that this definition of disease trajectory is similar to definitions of patient trajectories found in other literature (Pavalko, 1997; Pescosolido, 2013). Specifically, we take the first occurrence of a new disease code as it is recorded in the EHR.

\subsubsection{Disease Trajectory Modelling}

Disease trajectory models are typically represented as acyclic graphs, where each node represents a disease and each directed arc represents a progression from one disease to another. Reducing the graph to remove cycles presents a stronger representation of a general trajectory (progression) of diseases at the cost of simplifying the reality of complex real-world cases.

Constructing disease trajectories from EHR data is a challenging process. Various techniques have been used to investigate and model disease trajectories, including a data-driven approach (Glicksberg et al., 2016; Hanauer \& Ramakrishnan, 2013; Hidalgo et al., 2009; A. B. Jensen et al., 2014), data-mining (Giannoula et al., 2018; Ji et al., 2016; Wang et al., 2014), network-based (Steinhaeuser \& Chawla, 2009), free-text analysis (K. Jensen et al., 2017), and more recently by implementing a deeplearning techniques (Beaulieu-Jones et al., 2018; Futoma et al., 2015; Pham et al., 2017).

The disease trajectories modelled by A. B. Jensen et al. (2014) were constructed by joining overlapping pairs of diagnoses (bi-grams) to form longer trajectory chains. For example, the A. B. Jensen et al. method might have identified a bi-gram pair of diseases I21 (acute myocardial infarction) $\rightarrow$ I25 (chronic ischaemic heart disease), where I21 is a disease code that is recorded against a patient some time before I25. To construct a longer trajectory, A. B. Jensen et al. combined multiple bi-grams such as $\mathrm{I} 21 \rightarrow \mathrm{I} 25$ and I $25 \rightarrow \mathrm{N} 30$ to form a longer sequence of three diagnoses, $\mathrm{I} 21 \rightarrow \mathrm{I} 25 \rightarrow \mathrm{N} 30$. Although appealing in its simplicity, the concatenated trajectory might not be evidenced in any patient's record nor does it consider the conditional likelihood of the latter bigram given the former. No standard tools for disease trajectory modelling are evident in the literature. In contrast, process mining methods and tool are well established and have the potential to efficiently define such longer trajectories and also provide diagnostics to evaluate representativeness.

\subsection{Process Mining in Healthcare}

Process mining in healthcare is now well established with strong support from commercial and opensource tools, for example ProM Framework (Process Mining Group, 2010), Celonis (Celonis $\mathrm{GmbH}$, 2019), or Disco (Fluxicon BV, 2019). Process mining also boasts a growing body of literature (Rojas et al., 2016) and an international research community Process Oriented Data Science for Healthcare (“PODS4H," 2019).

The implementation of process mining in healthcare has been proven applicable to analyse care pathways (Mans et al., 2015; Rojas et al., 2016). Process mining is commonly used for mining the sequence of activities but the time between activities can also be analysed. Process mining has been used in cancer (Kurniati et al., 2016), cardiovascular disease (Kusuma et al., 2017), dentistry (Fox et al., 2018; R S Mans et al., 2012), frailty (Farid et al., 2019), sepsis (Mannhardt \& Blinde, 2017), and in primary care (Williams et al., 2018). Process mining is suitable for answering frequently posed questions by extracting information from an EHR (Mans et al., 2013). Unlike disease trajectory models which use the first occurrence of a disease, process mining is able to model multiple simultaneous and recurrent activity.

\section{MATERIALS AND METHOD}

The goal of this exploratory data-driven study is to explore the feasibility of producing disease trajectories using a process mining approach. This study uses synthetic data and has been made available on GitHub (Kusuma et al., 2019) and therefore useful for reproducibility. We examined A. B. Jensen et al.'s (2014) trajectories to simulate a set of EHR data that reflected a subset of the disease trajectories shown in Figure 1. Table 1 summarises the variables simulated that contained 50 patients with 146 diagnosis codes from 10 distinct diagnosis, using the first three characters of ICD-10 format. We treat each patient as a case and use the diagnosis codes in place of the standard process mining event-log activity names. 
The extracted data was formatted following the structure of a process mining event log, see Figure 2(a).

The synthetic event log was created by constructing event data for each case (patient) with the minimum of two events to form a diagnoses pair $(\mathrm{D} 1 \rightarrow \mathrm{D} 2)$. We created the time of D1 earlier than the time of D2, to ensure D1 occurred as an antecedent of D2. We created some cases with 3 or more events to represent a sequence of diagnoses $\mathrm{D} 1 \rightarrow \mathrm{D} 2 \rightarrow \mathrm{D} 3 \rightarrow \ldots$ which follow trajectories recognised by A. B. Jensen et al. as a collection of diagnoses pairs $(\mathrm{D} 1 \rightarrow \mathrm{D} 2$, $\mathrm{D} 2 \rightarrow \mathrm{D} 3, \mathrm{D} 3 \rightarrow \ldots$ ). To make the event log even look similar with the real-life EHR, then we added some repeating events as noise in the event log.

Table 1: The sources of required data from the synthetic dataset.

\begin{tabular}{|c|c|c|}
\hline Variables & Data & Field name \\
\hline $\begin{array}{l}\text { Case } \\
\text { identifier }\end{array}$ & Patient identifier & subject_id \\
\hline Event & Diagnosis code & diagnosis \\
\hline $\begin{array}{l}\text { Time } \\
\text { stamps }\end{array}$ & $\begin{array}{l}\text { Time when the } \\
\text { diagnosis was } \\
\text { recorded }\end{array}$ & admittime \\
\hline
\end{tabular}

For conducting the process mining experiments, we followed the Process Mining Project Methodology, $\mathrm{PM}^{2}$ (van Eck et al., 2015). Our use of the $\mathrm{PM}^{2}$ method is summarised below.

In Stage-1: Planning, research questions were identified from a literature review and confirmed by the project team during study planning. The team included a clinician, epidemiologist and computer scientists.

In Stage-2: Extraction, we defined the scope of the extraction by determining the granularity of the data, the time period, and selected the related attributes. We used the synthetic dataset as the input for creating an event log in the next stage. Only the first of any recurring diagnosis codes for each patient were used to create an acyclic, disease-trajectory model. We treated each patient as a case and used the diagnosis codes in place of the standard process mining event-log activity names.

In Stage-3: Data Processing, we followed the activities to create the event log as defined in $\mathrm{PM}^{2}$ by creating views, filtering logs, and event $\log$ transformation into pair log, the collection of event data in the form of pairs. The filtering was done by

\begin{tabular}{|ccc}
\hline Subject_id & Diagnosis & Time \\
\hline 3 & I21 & $01 / 01 / 2100$ \\
\hline 3 & I25 & $02 / 03 / 2100$ \\
3 & I25 & $12 / 05 / 2100$ \\
\hline 4 & I21 & $21 / 02 / 2100$ \\
diagnoses \\
4 & I25 & $14 / 06 / 2100$ \\
6 & I21 & $01 / 01 / 2100$ \\
6 & I25 & $02 / 01 / 2100$ \\
6 & J18 & $03 / 01 / 2100$ \\
\hline
\end{tabular}

(a)

\begin{tabular}{ccc}
\hline Subject_id & Diagnosis & Time \\
\hline 3 & I 21 & $01 / 01 / 2100$ \\
3 & I25 & $02 / 03 / 2100$ \\
4 & I21 & $21 / 02 / 2100$ \\
4 & I25 & $14 / 06 / 2100$ \\
6 & I 21 & $01 / 01 / 2100$ \\
6 & I25 & $02 / 01 / 2100$ \\
6 & J18 & $03 / 01 / 2100$ \\
\hline
\end{tabular}

(b)

\begin{tabular}{ccccc}
\hline Subject_id & Antecedent & Subsequent & Time1 & Time2 \\
\hline 3 & I21 & I25 & $01 / 01 / 2100$ & $02 / 03 / 2100$ \\
3 & I25 & I25 & $02 / 03 / 2100$ & $12 / 05 / 2100$ \\
4 & I21 & I25 & $21 / 02 / 2100$ & $14 / 06 / 2100$ \\
6 & I21 & I25 & $01 / 01 / 2100$ & $02 / 01 / 2100$ \\
6 & I25 & J18 & $02 / 01 / 2100$ & $03 / 01 / 2100$ \\
\hline
\end{tabular}

(c)

Figure 2: The filtering and transformation steps of event log: (a) the extracted event log from simulated data; (b) the recurrent diagnoses for each patient were filtered; and (c) the pair log with duplicate diagnoses removed.

removing the recurring diagnoses for each patient and keep the first occurrence.

In Stage-4: Mining and Analysis, the event log was analysed by applying process discovery and conformance-checking methods using process mining tools Disco and plugins in the ProM Framework. The discovered model was evaluated using the measures of fitness, precision and generalisability (van der Aalst, 2011). Fitness is a measure of how many traces in the event log can be replayed through the discovered model. Precision is a measure of how much the discovered model over estimates the traces in the event log; Low precision, or under-fitting, indicates that the model can represent traces that never occur in the event $\log$, while high precision, or over-fitting, indicates that the model can represent the traces in the event log, only. Generalisation is a measure of how often activities in the model occur in the event log.

Disease trajectories were 'discovered' using Disco, conformance-checking and the measurement of precision and generalisation were done in ProM Framework using the plugin Replay a Log on Petri 


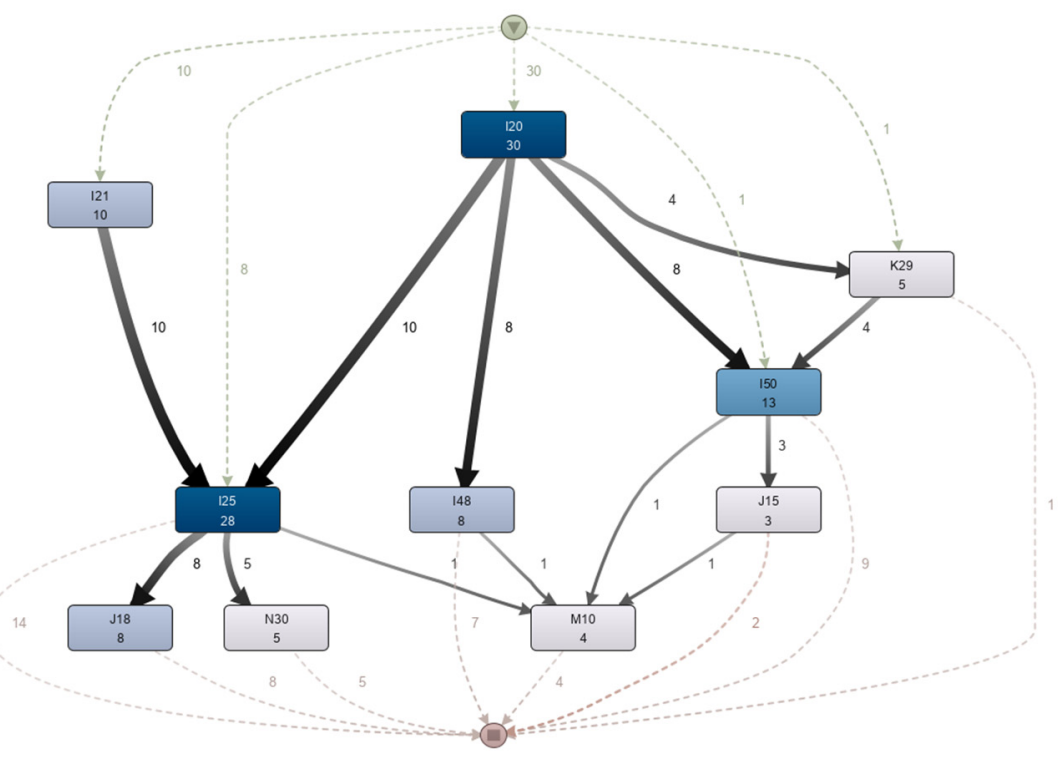

Figure 3: Disease trajectory model using process mining (the model generated from Disco).

Net for Conformance Analysis (Ardiansyah, 2012) and Measure Precision/Generalization respectively.

Because our discovered process model and the trajectories of A. B. Jensen et al. (2014) can both be considered directed graphs, we were able to assess agreement between them by checking if both were isomorphic. Two graphs are said to be isomorphic if they have the same ("iso-") structure ("-morph"), where structure is defined (Goldberg, 2003). In our case, by the count of diseases, the count of diseasepair connections and pattern of connected nodes and arcs is identical. More formally, two graphs are isomorphic if there exists a mapping that is a bijective function that preserves the branch structure of the graphs. We applied Cordella et al.'s (2001) method to check for isomorphism using the NetworkX Python library.

All processing other than discovery and conference checking were conducted in Python through Jupyter Notebook (Kluyver et al., 2016).

\section{RESULTS}

By following the $\mathrm{PM}^{2}$, the result of each stage is described. In Stage-1,we aimed to mine the disease trajectory agnostically without any specific selection of diagnosis and time window. We defined the main research question as Can disease trajectories be identified from an EHR, using a process mining approach?

In Stage-2, the synthetic data was formatted to follow the structure of a process mining event $\log$
(Figure 2a). In Stage-3, the recurring diagnoses for each patient were filtered out and we kept the first occurrence. The filtering step reduce the total number of events from 126 to 117 for the next stage.

In Stage-4, using the process-mining tool Disco a disease trajectory model was discovered (Figure 3). The trajectory model shows the same characteristics as the sub-trajectory of A. B. Jensen et al. (2014) in Figure 1. Both models have 10 nodes and 13 arcs including the thickness representation despite the difference on the scale and the addition of the case frequency (which is represented in Figure 3 by darker shades of nodes colour for more frequently occurring). The application of an isomorphic checker (following Cordella et al., 2001) determined that the two graphs could be considered isomorphic.

One benefit of our process-mining approach is that the temporal information about the time elapsed between disease occurrences is preserved and can be examined using standard tools. For example, the median duration between events can be displayed by process mining software such as Disco and ProM. The preservation of temporal data is a significant improvement over the simple models of disease sequence produced by the trajectory method of A, B. Jensen et al. (2014). Further, we can use process mining tools to measure the quality of the discovered model. In our experiment: the fitness value is 0.961 , precision 0.79 and generalisation 0.963 . 


\section{DISCUSSION}

In this work we sought to assess the feasibility of process mining to identify disease trajectories in a simulated dataset representative of a published disease trajectory. We applied the Process Mining Project Methodology $\mathrm{PM}^{2}$ to discover and conformance check a process model that was qualitatively similar to the sub-graph of trajectories from A. B. Jensen et al. (2014). In combination with good estimates for fitness, precision and generalisability, we conclude that process mining is a feasible approach for identifying disease trajectories using data similar to that found in EHRs.

The originality of this study is around the method where disease trajectories can be discovered using process mining techniques. We further suggest that our process mining method is an improvement on the disease trajectory method of A. B. Jensen et al. (2014). The high fitness, precision and generalisation scores permit us to make the follow statement: process models discovered using our methods on data similar to ours would permit the behaviour seen in the event $\log$, would be precise enough to not allow behaviour unrelated to what was seen in the event log, and would be general enough to reproduce future behaviour of the trajectories. This is in contrast to the concatenation of bi-grams approach of A. B. Jensen et al. (2014), which implies the existence of long trajectories of diseases based on combining direct disease pairs, end to end, without being in a position to validate from the data.

By default, process mining methods also provide additional information not found in the purelysequential output provided by A. B. Jensen et al. (2014). The output of some process mining algorithms present the durations and counts of cases that follow the trajectories. For example, the median duration among the patients is one day, while the longest duration is 150 days. Following our approach disease trajectory models can be automatically visualised in keeping with the graphical and exploratory ethos of the process mining. A major benefit of process mining is that its application is supported by commercial and open-source software (Fluxicon BV, 2019; Process Mining Group, 2010), a healthcare-specific literature base (Rojas, MunozGama, Sepúlveda, \& Capurro, 2016) and an international research community.

A limitation identified by our particular implementation of $\mathrm{PM}^{2}$ was the decision to include only the first occurrence of the primary diagnoses as the main event. This step promotes a representational bias that cannot be avoided in the study of model discovery. It is possible that different trajectories exist for recurrent diagnoses but we constrained our investigations for the purpose of demonstrating feasibility.

For future work, process mining should be applied to real-life EHRs to identify disease trajectories. Using high-volume datasets is necessary to evaluate could evaluate the scalability of the method. The role of a clinical domain expert could help to limit the number of variables of interest to build a more tightly-focussed model examining specific disease trajectory patterns and this approach would also make the discovery task more efficient. Despite the limitations, our approach has demonstrated that we can use process mining tools to mine disease trajectories and has opened up an interesting field for further work.

\section{CONCLUSION}

In this paper we have demonstrated the feasibility of mining of disease trajectories using process mining. The mining was conducted using a synthetic dataset which is similar to the data available from many EHR systems. Our study included the use of the $\mathrm{PM}^{2}$ framework to mine a representative disease trajectories model from EHR format data and addressed several quality dimension standards.

This feasibility study opens opportunities for future works in implementation the technique using population sized EHR data. The application of different discovery algorithms to mine the disease trajectory model may improve the conformance measurement and the disease trajectory model's quality dimension. Our approach will be of interest to the wide range of multi-disciplinary researchers interested in exploring healthcare record data for identifying disease trajectories to improve medicine and health.

\section{ACKNOWLEDGEMENTS}

This research is part of the first author's $\mathrm{PhD}$ study funded by the Indonesia Endowment Fund for Education (LPDP). The research was supported by the National Institute for Health Research (NIHR) Yorkshire and Humber Patient Safety Translational Research Centre (NIHR YH PSTRC). The views expressed in this article are those of the author(s) and not necessarily those of the NHS, the NIHR or the Department of Health and Social Care. 


\section{REFERENCES}

Ardiansyah, A. (2012). Replay a Log on Petri Net for Conformance Analysis-plugin.pdf.

Beaulieu-Jones, B. K., Orzechowski, P., \& Moore, J. H. (2018). Mapping patient trajectories using longitudinal extraction and deep learning in the MIMIC-III critical care database. Pacific Symposium on Biocomputing, O(212669), 123-132. https://doi.org/10.1142/978981 32355330012

Boyd, K. M. (2000). Disease, illness, sickness, health, healing and wholeness: Exploring some elusive concepts. Medical Humanities, 26(1), 9-17. https://doi.org/10.1136/mh.26.1.9

Casey, J. A., Schwartz, B. S., Stewart, W. F., \& Adler, N. E. (2016). Using Electronic Health Records for Population Health Research: A Review of Methods and Applications. Annual Review of Public Health, 37(1), 61-81. https://doi.org/10.1146/annurev-publhealth032315-021353

Celonis GmbH. (2019). Celonis. Retrieved from https://www.celonis.com/

Cordella, L. P., Foggia, P., Sansone, C., \& Vento, M. (2001). An improved algorithm for matching large graphs. 3rd IAPR-TC15 Workshop on Graph-Based Representations in Pattern Recognition, 149-159. Retrieved from https://citeseerx.ist.psu.edu/viewdoc/ summary?doi=10.1.1.101.5342

European Medicines Agency. (2002). Note for the Guidance on Good Clinical Practice. Retrieved from https://www.ema.europa.eu/en/documents/scientificguideline/ich-e6-r1-guideline-good-clinical-practice en.pdf

Farid, N., De Kamps, M., \& Johnson, O. (2019). Process Mining in Frail Elderly Care: A Literature Review. Biomedical Engineering Systems and Technologies, 5, 332-339. https://doi.org/10.5220/0007392903320339

Fluxicon BV. (2019). Disco. Retrieved from https://flux icon.com/disco/

Fox, F., Aggarwal, V. R., Whelton, H., \& Johnson, O. (2018). A data quality framework for process mining of electronic health record data. Proceedings - 2018 IEEE International Conference on Healthcare Informatics, ICHI 2018, 12-21. https://doi.org/10.1109/ICHI. 2018.00009

Futoma, J., Morris, J., \& Lucas, J. (2015). A comparison of models for predicting early hospital readmissions. Journal of Biomedical Informatics, 56, 229-238. https://doi.org/10.1016/j.jbi.2015.05.016

Giannoula, A., Gutierrez-Sacristán, A., Bravo, Á., Sanz, F., \& Furlong, L. I. (2018). Identifying temporal patterns in patient disease trajectories using dynamic time warping: A population-based study. Scientific Reports 2018 8:1, 8(1), 4216. https://doi.org/10.1038/s41598018-22578-1

Glicksberg, B. S., Li, L., Badgeley, M. A., Shameer, K., Kosoy, R., Beckmann, N. D., ... Dudley, J. T. (2016). Comparative analyses of population-scale phenomic data in electronic medical records reveal race-specific disease networks. Bioinformatics, 32(12), i101-i110. https://doi.org/10.1093/bioinformatics/btw282

Goldberg, M. (2003). The graph isomorphism problem. In J. L. Gross \& J. Yellen (Eds.), Handbook of graph theory (2nd ed., pp. 68-78). Boca Raton, FL: CRC Press.

Hanauer, D. A., \& Ramakrishnan, N. (2013). Modeling temporal relationships in large scale clinical associations. Journal of the American Medical Informatics Association, 20(2), 332-341. https://doi. org/10.1136/amiajnl-2012-001117

Hemingway, H., Asselbergs, F. W., Danesh, J., Dobson, R., Maniadakis, N., Maggioni, A., ... Denaxas, S. (2018). Big data from electronic health records for early and late translational cardiovascular research: Challenges and potential. European Heart Journal, 39(16), 14811495. https://doi.org/10.1093/eurheartj/ehx487

Hidalgo, C. A., Blumm, N., Barabási, A.-L., \& Christakis, N. A. (2009). A Dynamic Network Approach for the Study of Human Phenotypes. PLoS Computational Biology, 5(4), e1000353.

https://doi.org/10.1371/journal.pcbi.1000353

Jensen, A. B., Moseley, P. L., Oprea, T. I., Ellesøe, S. G., Eriksson, R., Schmock, H., ... Brunak, S. (2014). Temporal disease trajectories condensed from population-wide registry data covering 6.2 million patients. Nature Communications, 5(May), 1-10. https://doi.org/10.1038/ncomms5022

Jensen, K., Soguero-Ruiz, C., Oyvind Mikalsen, K., Lindsetmo, R. O., Kouskoumvekaki, I., Girolami, M., ... Magne Augestad, K. (2017). Analysis of free text in electronic health records for identification of cancer patient trajectories. Scientific Reports, 7. https://doi. org/10.1038/srep46226

Jensen, P. B., s, L. J., \& Brunak, S. (2012). Mining electronic health records: Towards better research applications and clinical care. Nature Reviews Genetics, Vol. 13, pp. 395-405. https://doi.org/ $10.1038 / \operatorname{nrg} 3208$

Ji, X., Chun, S. A., \& Geller, J. (2016). Predicting Comorbid Conditions and Trajectories Using Social Health Records. IEEE Transactions on Nano bioscience, 15(4), 371-379. https://doi.org/10.1109/ TNB.2016.2564299

Kluyver, T., Ragan-kelley, B., Pérez, F., Granger, B., Bussonnier, M., Frederic, J., ... Willing, C. (2016). Jupyter Notebooks - a publishing format for reproducible computational workflows. In Positioning and Power in Academic Publishing: Players, Agents and Agendas. https://doi.org/10.3233/978-1-61499. 649-1-87

Kurniati, A. P., Johnson, O., Hogg, D., \& Hall, G. (2016). Process Mining in Oncology: a Literature Review. Information Communication and Management (ICICM). https://doi.org/10.1109/INFOCOMAN. 2016.7784260

Kusuma, G., Bennett, B., \& Johnson, O. (2017). Process analysis in cardiovascular disease using process mining. Abstract 621 in Scott P.J. et Al. Journal of Innovation in Health Informatics, 24(1), 171. https://doi.org/10.14236/jhi.v24i1.939 
Kusuma, G., Sykes, S., McInerney, C., \& Johnson, O. (2019). Resource of Process Mining for Disease Trajectory Mining. Retrieved from https:/github.com/ gpkusuma/dtm_processmining

Mannhardt, F., \& Blinde, D. (2017). Analyzing the trajectories of patients with sepsis using process mining (Vol. 1859). Retrieved from APA website: www. tue.nl/taverne

Mans, R. S., Reijers, H. A., Van Genuchten, M., \& Wismeijer, D. (2012). Mining processes in dentistry. IHI'12 - Proceedings of the 2nd ACM SIGHIT International Health Informatics Symposium, 379-388. https://doi.org/10.1145/2110363.2110407

Mans, R. S., van der Aalst, W. M. P., \& Vanwersch, R. J. B. (2015). Process Mining in Healthcare Evaluating and Exploiting Operational Healthcare Processes. https://doi.org/10.1007/978-3-319-16071-9

Mans, R. S., van der Aalst, W. M. P., Vanwersch, R. J. B., \& Moleman, A. J. (2013). Process Mining in Healthcare: Data Challenges when Answering Frequently Posed Questions. In Lenz R., Miksch S., Peleg M., Reichert M., Riaño D., \& ten Teije A. (Eds.), Lecture Notes in Computer Science (Vol. 7738, pp. 140-153). Retrieved from http://wwwis.win. tue.nl/ wvdaalst/publications/p707.pdf

Muhrer, J. C. (2014). The importance of the history and physical in diagnosis. The Nurse Practitioner, 39(4), 30-35. https://doi.org/10.1097/01.NPR.0000444648.20444.e6

Murray, S. A., Kendall, M., Boyd, K., \& Sheikh, A. (2005). Illness trajectories and palliative care. $B M J, 330(7498)$, 1007-1011. https://doi.org/10.1136/bmj.330.7498.100 7

Pavalko, E. K. (1997). Beyond Trajectories: Multiple Concepts for Analyzing Long Term Process. In M. A. Hardy (Ed.), Studying Aging and Social Change: Conceptual and Methodological Issues (pp. 129-147). Thousand Oaks, CA: SAGE Publications Ltd.

Pescosolido, B. A. (2013). Patient Trajectories. In The Wiley Blackwell Encyclopedia of Health, Illness, Behavior, and Society (pp. 1770-1777). https://doi.org/10.1002/9781118410868.wbehibs 282

Pham, T., Tran, T., Phung, D., \& Venkatesh, S. (2017). Predicting healthcare trajectories from medical records: A deep learning approach. Journal of Biomedical Informatics, 69, 218-229. https://doi.org/10.1016/ j.jbi.2017.04.001

PODS4H. (2019). Retrieved from http://pods4h.com

Process Mining Group. (2010). ProM - Process Mining Toolkit. Retrieved from www.promtools.org

Rojas, E., Munoz-Gama, J., Sepulveda, M., \& Capurro, D. (2016). Process mining in healthcare: A literature review. Journal of Biomedical Informatics, 61(April), 224-236. https://doi.org/10.1016/j.jbi.2016.04.007

Rothman, K. J., \& Greenland, S. (2005). Causation and causal inference in epidemiology. American Journal of Public Health, 95(SUPPL. 1), S144-50. https://doi.org/10.2105/AJPH.2004.059204

Steinhaeuser, K., \& Chawla, N. V. (2009). A networkbased approach to understanding and predicting diseases. Social Computing and Behavioral Modeling, 209-216. https://doi.org/10.1007/978-1-4419-0056-226

Sumida, K., \& Kovesdy, C. P. (2017). Disease Trajectories Before ESRD: Implications for Clinical Management. Seminars in Nephrology, 37(2), 132-143. https://doi. org/10.1016/j.semnephrol.2016.12.003

van der Aalst, W. M. P. (2011). Process Mining: Discovery, Conformance and Enhancement of Business Processes. https://doi.org/10.1007/978-3-642-19345-3

van Eck, M. L., Lu, X., Leemans, S. J. J., \& van Der Aalst, W. M. P. (2015). PM2: A process mining project methodology. In J. Zdravkovic, M. Kirikova, \& P. Johannesson (Eds.), Lecture Notes in Computer Science (including subseries Lecture Notes in Artificial Intelligence and Lecture Notes in Bioinformatics) (Vol. 9097, pp. 297-313). https://doi.org/10.1007/978-3319-19069-3 19

Wang, X., Sontag, D., \& Wang, F. (2014). Unsupervised Learning of Disease Progression Models. https://doi.org/10.1145/2623330.2623754

WHO. (2019). Classification of Diseases. Retrieved from http://www.who.int/classifications/icd/en/

Williams, R., Rojas, E., Peek, N., \& Johnson, O. A. (2018). Process mining in primary care: A literature review. Studies in Health Technology and Informatics, 247, 376-380. https://doi.org/10.3233/978-1-61499-852-5376

World Health Organization. (2002). Medical Records Manual : A Guide for Developing Countries. Retrieved from https://apps.who.int/iris/bitstream/handle/10665/ 208125/9290610050_rev_eng.pdf?sequence $=1 \&$ isAllo wed $=\mathrm{y}$

World Health Organization. (2016). Diagnostic errors: technical series on safer primary care. Retrieved from http://apps.who.int/bookorders 